\title{
Association of Metabolic Obesity Phenotypes and Total Testosterone in Middle-aged and Elderly Male Population
}

\section{Qingbo Guan ( $\nabla$ doctorguanqingbo@163.com )}

Department of Endocrinology, Shandong Provincial Hospital, Cheeloo College of Medicine, Shandong University, Jinan, Shandong, 250021, China. https://orcid.org/0000-0002-1549-5304

\section{Luna Liu}

Shandong provincial hospital

Shuang Liu

Shandong Provincial hospital

Qianmei Song

Shandong provincial hospital

Dandan Luo

Shandong Provincial hospital

\section{Yu Su}

Shandong provincial hospital

\section{Xiangyu Qi}

Shandong provincial hospital

\section{Qian Wang}

Shandong provincial hospital

Jing Ning

Shandong Provincial Hospital

\section{Youyuan LV}

Shandong Provincial Hospital

\section{Research}

Keywords: metabolic obesity phenotypes, testosterone, metabolically healthy obese, metabolically unhealthy normal weight, hypotestosteronemia

Posted Date: November 20th, 2020

DOl: https://doi.org/10.21203/rs.3.rs-108424/v1 
License: (c) (i) This work is licensed under a Creative Commons Attribution 4.0 International License. Read Full License 


\section{Abstract}

Objectives Obesity and metabolic syndrome have been reported to exert an impact on male reproductive system with decreasing levels of serum total testosterone (TT), however, the effect of different metabolic obesity phenotypes on testosterone has been poorly studied. We aimed to evaluate the association of metabolic obesity phenotypes and total testosterone levels in male population.

Methods We performed a retrospective study based on an epidemiological investigation, a total of 4081 middle-aged and elderly male individuals were recruited. The population was classified as metabolically healthy normal weight (MHNW); metabolically healthy obese (MHO); metabolically unhealthy normal weight (MUNW) and metabolically unhealthy obese (MUO) according to normal weight (BML $<25.0)$ and obesity (BMI $\geq 25.0$ ) with or without metabolic syndrome.

Results We collected 563 hypotestosteronemia among 4081 middle-aged and elderly male individuals. The odds ratios (ORs) (95\% Cls) of hypotestosteronemia in obesity and metabolic syndrome was 3.072 (2.414-3.911) and 3.294 (2.631-4.125) respectively, after adjusting for age, luteinizing hormone, smoking status and alcohol consumption. Compared to MHNW group, male subjects in MHO, MUNW and MUO groups had a decreased serum TT levels. Additionally, MUO group have a lowest concentration of serum TT and a highest proportion of hypotestosteronemia. There was no significant difference of TT levels between $\mathrm{MHO}$ and MUNW group.

Conclusions Obesity and metabolic syndrome are independent risk factor of hypotestosteronemia in middle-aged and elderly male populations. Our study also suggested that individuals with MHO, MUNW and MUO have a higher risk of developing hypotestosteronemia.

\section{Introduction}

Obesity is an important chronic disease all over the world because of the increasing prevalence and high risk for public health. Obesity can result in multiple alterations in the endocrine systems, including altered circulating blood hormone concentrations, because of changes in the pattern of secretion and/or metabolism, abnormal hormonal transport, and/or action at levels of target tissues [1]. It has been demonstrated that obesity has an impact on male reproductive system. And obesity has been considered a risk for male hypotestosteronemia with low testosterone levels. It has been reported that there are $20 \%$ to $64 \%$ of obese men with low total testosterone (TT) or free testosterone (FT) levels [2]. Related studies have reported that the increasing BMI is associated with low testosterone and sex-hormone-bindingglobulin (SHBG) levels in male population [3-6]. Weight reduction by lifestyle intervention or bariatric surgery can increase the levels of TT and SHBG [3].

Metabolic syndrome (MS), gathering of abdominal obesity, hypertension, hyperglycemia and dyslipidemia is an important challenge for health problem. The prevalence of metabolic syndrome ranges from 10 to 34 percent according to different definitions or populations [7]. Several studies have pointed that metabolic syndrome can be regarded as an independent association with low levels of total 
testosterone in male population [8-11]. And low levels TT and SHBG can be considered as independent risk factors for metabolic syndrome [12-13]. However, the relationship of TT with metabolic syndrome remains inconsistent. A cross-sectional study reported that there was no significant difference of total testosterone among MS and non-metabolic syndrome group. It is supposed that the more parameters are abnormal, the lower levels of SHBG would be measured, without alternations of TT [14]. And several studies have concluded that their data were not consistent with the hypothesis that low levels of TT were associated with incidence of metabolic syndrome [15-16].

Recent studies have demonstrated that not all obesity individuals have metabolic abnormal risk factors, and not all lean individuals are metabolic healthy [17]. Therefore, different metabolic phenotypes of obesity attract researchers' attention in recent years. Although obesity may induce decreased serum TT concentration by some related mechanisms, the fact is that only a small proportion of obese men are hypotestosteronemia, probably those genetically predisposed or morbidly obese [18]. Metabolic healthy obesity ( $\mathrm{MHO}$ ) is considered to be obesity (BMI $\geqq 25 \mathrm{~kg} / \mathrm{m}^{2}$ ) without metabolic diseases (hypertension, type 2 diabetes mellitus (T2DM) and dyslipidemia). In addition, metabolically unhealthy obesity (MUO) refers to a proportion of obesity subjects with metabolic diseases. A recent study based on 4945 men with sexual dysfunction and 231 male partners of infertile couples demonstrated that men in $\mathrm{MHO}$ groups had lower total testosterone levels compared to men with normal weight [19]. However, the effect of specific metabolic obesity phenotypes on total testosterone in general male population remains unknown. Hypotestosteronemia classified into primary hypotestosteronemia which results from testicular defects and is associated with low testosterone levels and elevated LH and FSH levels and secondary hypotestosteronemia which results from dysfunction of hypothalamus and/or pituitary and refers to patients with low testosterone levels and low or inappropriately normal LH and FSH levels. And whether the influence of different metabolic obesity phenotypes on TT levels is caused by affecting hypothalamus and/or pituitary levels or testicular levels remains unknown.

Thus, we performed the study to verify the influence of MS and obesity separately on male hypotestosteronemia, and investigate the relationship of different metabolic obesity phenotypes and male total testosterone levels in a large-scale male population.

\section{Subjects And Methods}

\section{Subjects}

We recruited nearly 11,000 persons participated in the population-based cross-sectional study in Ningyang County (Taian, Shandong Province, China) from June to November 2011. All participants were local-registered residents aged 40 years and older who have lived there for at least 5 years. All participants were asked to complete a self-reported questionnaire and provided an overnight fasting blood sample. In our study, the exclusion criteria were as follows: i) female; ii) no information on vital statistics (such as age, sex, height or weight) or missing data on serum total testosterone (TT), blood pressure, fasting serum glucose or lipid levels; iii) taking medications that might affect TT level (such as 
androgens, steroid hormones); and iv) severe hepatic or renal disorders, lung diseases, hypothalamus and/or pituitary gland diseases, neurologic diseases, or tumors that might affect TT level (such as brain cancer or prostate cancer). At last, a total of 4,081 participants were recruited in this study. We retrospectively reviewed the patients' medical records for research purposes only, which was approved by the Ethics Committee of Shandong Provincial Hospital affiliated to Shandong University.

\section{Data and specimen collection}

Height and WC were measured adjusting by $0.1 \mathrm{~cm}$. Weight was measured adjusting by $0.1 \mathrm{~kg}$. BMI was obtained by dividing weight in kilograms by the square of height in meters. The average of the three blood pressure measurements in sitting position after a 5-minute rest was used in the analysis. Past medical history, smoking status and alcohol consumption were obtained by a questionnaire. The diseases (hypertension, diabetes mellitus, and coronary heart disease) were based on previous diagnosis by a physician. Smoking status and alcohol consumption were defined as never, ever and current.

Venous blood samples were collected from all patients after at least 10-h overnight fast. And samples were separated and preserved in $-80^{\circ} \mathrm{C}$. The concentrations of total cholesterol (TC), triglyceride (TG), high density lipoprotein cholesterol (HDL-C), fasting plasma glucose (FPG) and glycosylated hemoglobin (HbA1c) were measured directly with an ARCHITECT ci16200 Integrated System (Abbott). Serum TT, luteinizing hormone (LH) and follicle-stimulating hormone (FSH) were measured using electrochemiluminescent procedures (Cobas E601; Roche) at the clinical laboratory of Shandong Provincial Hospital.

\section{Study outcome definition}

Total testosterone level $<12 \mathrm{nmol} / \mathrm{L}$ is suggestive of hypotestosteronemia [20]. Secondary hypotestosteronemia refers to patients with low testosterone levels and low or inappropriately normal LH and FSH levels. Primary hypotestosteronemia is associated with low testosterone levels and elevated $\mathrm{LH}$ ( $>8.6 \mathrm{mlU} / \mathrm{L})$ and FSH (>12.4 mIU/L) levels. We used the Adult Treatment Panel $\otimes$ definition of metabolic syndrome in male population: having three or more following criteria: waist circumference $>102 \mathrm{~cm}$, triglycerides $>1.7 \mathrm{mmol} / \mathrm{L}$ (or treatment for hyperlipidemia), $\mathrm{HDL}-\mathrm{C}<1 \mathrm{mmol} / \mathrm{L}$, plasma fasting glucose $>100 \mathrm{mg} / \mathrm{dl}$, and systolic/diastolic blood pressure $>130 / 85 \mathrm{mmHg}$ (or treatment for hypertension) [21]. And we classified the population as normal weight (BML25.0) and obesity $(B M I \geq 25.0)$ with or without metabolic syndrome. Then the participants were classified as four groups: metabolically healthy normal weight (MHNW); metabolically healthy obese (MHO); metabolically unhealthy normal weight (MUNW) and metabolically unhealthy obese (MUO).

\section{Statistical analysis}

SPSS22.0 software was used for the statistical analysis. Continuous variables were presented as mean \pm standard deviations, and between-group differences were assessed by a single-factor analysis of variance (ANOVA). Categorical variables were presented as percentage, and between-group differences 
were assessed by Chi-squared test. Multiple logistic regression analysis was performed to assess the risk factors of hypotestosteronemia. All P-values were 2-sided, and less than 0.05 were considered statistically significant.

\section{Results}

\section{Baseline characteristics of participants in different metabolic obesity phenotypes}

A total of 4081 male participants aging from 40 to 75 years were enrolled into the final analysis. There were 563 participants suffering from hypotestosteronemia (13.80\%). Within metabolic categories, obese men tended to be younger with a high level of $\mathrm{BMI}$ and WC than male normal weight. And obese men were more likely to have a high level of TC, TG, LDC-C, ALT, GGT, DBP and a low level of HDL-C, TT, LH and $\mathrm{FSH}$, without difference of AST, FBG and HbA1C. As expected, compared to metabolic healthy group, metabolic unhealthy patients were more likely to have a high level of TG, TC, ALT, AST, GGT, FPG, HbA1C, systolic blood pressure and diastolic blood pressure and a low level of HDL-C and TT. There was high prevalence of diabetes mellitus, hypertension and coronary heart disease in metabolic unhealthy population. Compared to MHNW group, MHO, MUNW and MUO group tended to have a high level of BMI, WC, elevated liver function index, altered metabolic syndrome components and high risk of development of hypertension and coronary heart disease, as well as decreased levels of serum TT, LH and FSH (Tab 1). There was no significant difference of serum testosterone level between $\mathrm{MHO}$ and MUNW group. The significant differences in the composition of the four groups with respect to smoking status and alcohol consumption were also shown in Tab 1.

\section{The prevalence of hypotestosteronemia in different metabolic obesity phenotypes}

To compare the difference of prevalence of hypotestosteronemia among different metabolic obesity phenotypes, we performed Chi-squared test between each two groups. We observed obvious elevated trend of prevalence of hypotestosteronemia in these four metabolic obesity phenotypes in Fig 1. Compared to MHNW group, other three groups have been demonstrated to have a higher risk of hypotestosteronemia. At the same time, compared to MUO group, other three groups were more likely to have a lower risk of hypotestosteronemia. However, no difference existence between MUNW and MHO group (Fig 1). The results of comparison of prevalence of secondary hypotestosteronemia or primary hypotestosteronemia in four groups were similar to hypotestosteronemia. However, the difference of prevalence of primary hypotestosteronemia in $\mathrm{MHO}$ and $\mathrm{MUO}$ groups was not significant. These differences demonstrated that different metabolic obesity phenotypes could have a different impact on serum testosterone levels.

\section{The separated and synergistic effect of obesity and metabolic status on serum TT concentration}

To examine the separated and synergistic effect of obesity and metabolic status on serum TT concentration, we carried out multivariate logistic regression analysis of hypotestosteronemia with low serum TT levels ( $<12 \mathrm{nmol} / \mathrm{I})$ adjusting for age, LH, smoking status (never, ever, current) and alcohol 
intake (never, ever, current). Both obesity and metabolic syndrome, considered separately, were risk factor of low serum TT levels. Multivariate-adjusted Odd ratio (OR) of low serum TT levels in obesity men was 3.072 (95\% Cl: 2.414-3.911). The risk of low serum TT levels increased appropriately 3.294-fold in metabolic syndrome individuals compared to metabolic healthy individuals. Although the risk of obesity and metabolic syndrome were attenuated after mutual analysis, the OR of low serum TT levels remained statistically significant adjusting for age, LH, smoking status (never, ever, current) and alcohol intake (never, ever, current).

\section{The independence of different metabolic obesity phenotypes for hypotestosteronemia}

To study the independence of different metabolic obesity phenotypes for male hypotestosteronemia, we performed logistic regression analysis to observe the OR of hypotestosteronemia in four groups. Compared to participants in MHNW group, the risk of developing hypotestosteronemia in MHO, MUNW and MUO groups significantly increased. OR of hypotestosteronemia in MHO, MUNW and MUO groups was 2.736 (95\% Cl:2.132-3.512), 3.332 (2.314-4.799) and 6.213 (4.842-7.972), respectively. After adjusted for age, OR of hypotestosteronemia in $\mathrm{MHO}$ and MUO groups decreased, but it was still significant. Male subjects in MHO, MUNW and MUO also had a significantly increased risk of hypotestosteronemia in contrast to MHO group after adjusted for age, LH, smoking status (never, ever, current) and alcohol intake (never, ever, current).

\section{Discussion}

Obesity and metabolic syndrome are all considered to be risk factor of low serum TT levels. We provided precise insight into the association between different metabolic obesity phenotypes and low serum TT levels in a large-scale population of Chinese middle-aged and elderly men. It demonstrated that different metabolic obesity phenotypes including MHO, MUNW and MUO can both increase the risk of low serum TT levels, especially significantly in MUO group.

In our study, we draw a conclusion that both obese men and metabolic unhealthy men had a higher risk of developing low serum TT levels than normal weight and metabolic healthy individuals, which is consistent with the results in previous study [8-11, 13]. It demonstrated that serum TT levels in male population were associated with BMI and different components of metabolic syndrome. A Korean study have shown that serum TT levels was negatively associated with BMI, WC, FPG, TG and blood pressure, and positively associated with HDL-L levels [11]. A meta-analysis that included 9,525 men of different ethnics proved that serum TT levels were negatively correlated with each component of metabolic syndrome [8]. It has been reported that hyperinsulinemia which was associated with metabolic syndrome can inhibit the secretion of testosterone through insulin receptors which were expressed on Leydig cells [22]. And excess adiposity tissue could lead to high levels of leptin, which can inhibit the steroidogenesis and the secretion of testosterone. Inflammatory cytokines, specially IL-1 $\beta$, IL- 6 and TNF- $a$, which were elevated in obesity or metabolic unhealthy individuals, can directly inhibit the steroidogenesis and subsequent testosterone production [23]. Although there are several studies investigating the association 
of obesity and metabolic syndrome components with testosterone levels separately, there is still quite a gap in the literature considering the relationship of different metabolic obesity phenotypes with total testosterone levels.

As we have proved, different metabolic obesity phenotypes except for MHNW group are at a high risk of developing low serum TT levels. Among these groups, MUO individuals are more likely to have a low level of serum TT than others. Studies have demonstrated that age, smoking status and alcohol consumption were associated with different metabolic obesity phenotypes [24,25]. However, after adjusting these above confounding factors, the relationship between TT levels and metabolic obesity phenotypes remained significant. To the best of our knowledge, the only study on this topic was performed and explored male sexual dysfunction with different metabolic obesity phenotypes [19]. It showed that the men with $\mathrm{MHO}$ and metabolically complicated obesity (MCO) had lower testosterone levels and only MCO men had worse erectile function compared with normal weight men. However, the results were derived from male patients with sexual dysfunction or infertility, which could have different characteristics with the general male population. What's more, the definition of MCO was the presence of at least 1 abnormality among hypertension, low HDL-C and diabetes in obese men, and the contrast group was men with normal weight regardless of metabolic status.

In our study, there was a higher risk of developing hypotestosteronemia in male patients in $\mathrm{MHO}$ compared to MHNW group, but a lower risk than MUO group. MHO individuals referred to obese people without the risk factor of metabolic syndrome, and several studies have reported that these individuals were fat but fit with a more favorable inflammatory and metabolic profiles [26]. However, the recent systematic review and meta-analysis showed that MHO subjects had an increased risk for the development of cardiovascular diseases and all-cause mortality in contrast to MHNW subjects [27]. And our results indicated that $\mathrm{MHO}$ individuals had a decreased total testosterone level compared to MHNW individuals. It has been reported that metabolic healthy obese individuals have certain difference in multiple aspects, such as, fat distribution, inflammatory status, insulin resistance and postprandial lipaemia, compared with metabolic unhealthy obese [28,29]. Compared with MUO individuals, MHO was shown to have increased subcutaneous fat relative to visceral fat, lower liver fat and insulin sensitivity. Excessive visceral fat or insulin resistance rather than general adiposity in obese individuals were high risk of pre-diabetes and type 2 diabetes mellitus. Metabolic healthy subjects displayed lower postprandial response of plasma TG and hs-CRP, compared with those metabolic unhealthy, independently whether or not they were obese. We also observed that testosterone levels were decreased in male subjects with MUO compared to MHO. Clinical features and metabolism difference caused by metabolic healthy obesity phenotype can partly explain the alternations of testosterone concentration in $\mathrm{MHO}$ group compared to MHNW and MUO group. Since MHO is a dynamic state (with a significant proportion of $\mathrm{MHO}$ subjects progressing to MUO over time), it is necessary to encourage weight control in $\mathrm{MHO}$ phenotypes to prevent the development of metabolic related disease including hypotestosteronemia.

We also observed that testosterone levels in male subjects with MUNW were decreased compared to MHNW group, but these were increased in MUNW group than MUO group, and no significant difference to 
MHO group. MUNW individuals displayed several metabolic abnormal risks including insulin resistance, atherogenic lipid profiles, visceral adiposity accumulation and lower physical energy expenditures, despite having a normal $\mathrm{BMI}$, and eventually had the increased risk of cardiovascular disease [30]. It has been reported that MUNW phenotype exhibited increased arterial stiffness and carotid atherosclerosis compared to MHNW or MHO subjects [31]. And another report has shown that MUNW individuals had a higher risk of cardiovascular disease than $\mathrm{MHO}$, as well as reduced morbidity and mortality [32]. The $\mathrm{MHO}$ phenotype was associated with a better overall metabolic profile and less oxidative stress than that observed in MUNW individuals [33]. In our study, there is no significant difference of testosterone levels between MUNW and MHO group, which indicated that both MUNW and $\mathrm{MHO}$ can exert an influence on total testosterone levels. The underlying mechanism needs further study. It was shown that male subjects in MUO group have a decreased serum TT levels compared to MHO and MUNW groups, which can be explained by synergistic effect of obesity and metabolic unhealthy on TT levels in patients with MUO. It also indicated that male patients with $\mathrm{MHO}$ or MUNW should pay attention to losing weight or improving metabolic status in order to prevent the further drop of TT levels.

It is well known that testosterones are mainly synthesized in Leydig cell, which could be modulated by hypothalamus-pituitary levels. In our study, we also found that gonadotropins FSH and LH was significantly reduced in $\mathrm{MHO}$ and $\mathrm{MUO}$ group compared to $\mathrm{MHNW}$. However, there was no significant difference of FSH and LH levels between MHNW and MUNW, as well as MHO and MUO group. There was an increasing conversion of androgen precursors into estrogens by aromatase system in adipose tissue among obese male population. Inappropriate effects of increasing estrogens might reduce LH secretory mass per secretory burst without any alternation in burst number [34]. The exact mechanism for decreased gonadotrophins in male obesity could also include leptin, inflammatory mediators and hypothalamic kisspeptin affecting gonadotropins- releasing hormone secretion [35]. These results indicated that low levels of gonadotropins can partly explain the decreasing total testosterone levels in obesity participants, but not affected by metabolic status. To observe the effect of metabolic obesity phenotypes on pituitary or testicular levels, we further compared the difference of prevalence of secondary hypotestosteronemia and primary hypotestosteronemia in four metabolic obesity phenotypes. It showed that the difference of prevalence of secondary hypotestosteronemia and primary hypotestosteronemia in four groups was similar to hypotestosteronemia. The results were confusing, which was inconsistent with the results that LH and FSH levels were only affected by obesity. Although we didn't get information about the pulse and frequency of LH and FSH secretory, which also played important role in regulating the production of testosterone levels. Therefore, further studies about the pulse and frequency of LH and FSH secretory, the alternations of structure and function in testis are needed to perform in different metabolic obesity phenotypes.

This is the first study to investigate the relation of different metabolic obesity phenotypes with serum TT concentration in a large-scale male population. In our study, other potential risk factors of low TT levels such as age, LH levels, smoking status and alcohol consumption were taken into account to make the result more convincing. However, several limitations also existed in our study. We didn't obtain information about other sex hormone such as SHBG, estrogen, which can have an effect on TT levels. 
Meanwhile, we can't draw a conclusion about the association about different metabolic obesity phenotypes and other sex hormones. What's more, the cross-sectional study was unable to detect any causal relationship between different metabolic obesity phenotypes and testosterone levels. Large prospective studies are needed to validate this relationship.

In conclusion, both obese men and metabolic unhealthy men have a higher risk of developing low serum TT levels. Different metabolic obesity phenotypes groups have influence on lower serum testosterone level in different degree, especially in MUO group with a lowest serum TT concentration. Male subjects in $\mathrm{MHO}$ and MUNW groups had significantly decreased testosterone levels compared to MHNW group, however, there was no significant difference of testosterone levels between MUO and MUNW groups. Clinical physicians should pay attention to the weight combined with metabolic status of patients when we explored the reason of decreasing concentration of serum TT in male populations. Individuals weight control and prevention of metabolic syndrome may be used for the primary prevention of male hypotestosteronemia. Patients with low level of serum TT can get improved by losing weight or improving metabolic status.

\section{Declarations}

\section{Acknowledgements:}

The research used data collected as part of a IONgitudinal study (REACTION) which was a multi-center, prospective, observational cohort study in middle-aged to elderly Chinese people. We gratefully acknowledge the REACTION study group for organizing the national epidemiological REACTION study in China.

\section{Author's contribution:}

Author contributions included the following: L.L. analyzed the data and wrote the manuscript. S.L., Q.S. and X.Q. contributed to clinical data collection. D.L. and Y.S. conducted the research. Q.W., Y. L. and J.N. organized and analyzed the data. Q.G. designed and performed the study and are responsible for the data.

\section{Availability of data:}

The data used to support the findings of this study are available from the corresponding author or first author upon request.

\section{Funding:}

This work was supported in part by grants from the National Natural Science Foundation of China (81770860, 81471078 and 81641030) and Key Research and Development Plan of Shandong Province (2016GSF201007). 


\section{Ethics approval and consent to participate:}

We retrospectively reviewed the patients' medical records for research purposes only, which was approved by the Ethics Committee of Shandong Provincial Hospital affiliated to Shandong University.

\section{Consent for publication}

Not applicable.

\section{Competing interest:}

The authors declare that there is no conflict of interests.

\section{Data Available Statement}

The data used to support the findings of this study are available from the corresponding author or first author upon request.

\section{References}

1. Pinkey JH, Kopelman PG. Endocrine determinant of obesity. In: Bray GA, Bouchard C, Handbook of obesity. Etiology and pathophysiology. 2nd ed. New York: Marcel Dekker; 2004. p. 655-70.

2. Kalyani RR, Dobs AS. Androgen deficiency, diabetes, and the metabolic syndrome in men. Curr Opin Endocrinol Diabetes Obes. 2007;14:226-234.

3. Hector F. Escobar-Morreale, Elisa Santacruz, Manuel Luque-Ramírez, and José I. Botella Carretero. Prevalence of 'obesity-associated gonadal dysfunction' in severely obese men and women and its resolution after bariatric surgery: a systematic review and meta-analysis. Human Reproduction Update. 2017;1-19.

4. Pasquali, V. Vicennati, and A. Gambineri. Adrenal and gonadal function in obesity. J. Endocrinol. Invest. 2002; 25: 893-898.

5. Eriksson J, Haring R, Grarup N, Vandenput L, Wallaschofski H, Lorentzen E, et al. Causal relationship between obesity and serum testosterone status in men: A bi-directional mendelian randomization analysis. PLOS ONE 2017;12(4).

6. Renato Pasquali, M.D. Obesity and androgens: facts and perspectives. Fertility and Sterility. 2006;85:1319-40.

7. Munoz-Garach A, Cornejo-Pareja I, Tinahones FJ. Does metabolically healthy obesity exist? Nutrients 2016;8(6):320.

8. Brand, J.S., Brand JS, Rovers MM, Yeap BB, Schneider HJ, Tuomainen TP, Haring R, et al. Testosterone, sex hormone-binding globulin and the metabolic syndrome in men: an individual participant data meta-analysis of observational studies. PLoS One. 2014 Jul 14;9(7):e100409. 
9. Corona G, Monami M, Rastrelli G, Aversa A, Tishova Y, Saad F, et al. Testosterone and metabolic syndrome: a meta-analysis study. J Sex Med. 2011;8(1):272-83.

10. Petya Angelova, Zdravko Kamenov, Adelina Tsakova, Yosif El-Darawish, Haruki Okamura. Interleukin18 and testosterone levels in men with metabolic syndrome. THE AGING MALE, 2017.

11. Doohee Hong, Young-Sang Kim, Eun Soo Son, Kyu-Nam Kim, Bom-Taeck Kim, Duck-Joo Lee, et al. Total testosterone and sex hormone-binding globulin are associated with metabolic syndrome independent of age and body mass index in Korean men. Maturitas .2013;74:148-153.

12. Emmanuela Quental Callou de Sá, Francisco Carleial Feijó de Sá, Kelly Cristina Oliveira, Fausto Feres, leda Therezinha Nascimento Verreschi. Association between sex hormone-binding globulin (SHBG) and metabolic syndrome among men. Sao Paulo Med J. 2014; 132(2):111-5.

13. Zhang J, Huang X, Liao M, Gao Y, Tan A, Yang X, et al. Both total testosterone and sex hormonebinding globulin are independent risk factors for metabolic syndrome: results from Fangchenggang Area Male Health and Examination Survey in China. Diabetes Metab Res Rev 2013; 29: 391-397.

14. Khalid Siddiqui1, Khalid Al-Rubeaan, Shaik Sarfaraz Nawaz, Khaled H. Aburisheh, Anas M. Zein Alaabdin, Ibrahim A. Tolba. Serum Sex Hormone Binding Globulin (SHBG) Relation with Different Components of Metabolic Syndrome in Men with Type 2 Diabetes. Horm Metab Res.

15. Callou de Sá EQ, Feijó de Sá FC, e Silva R de S, Kelly Cristina de Oliveira, Alexis Dourado Guedes, Fausto Feres, et al. Endogenous oestradiol but not testosterone is related to coronary artery disease in men. Clin Endocrinol (Oxf). 2011;75(2):177-83.

16. Bhasin S, Jasjua GK, Pencina M, Ralphd'Agostino, SR., Andread. Coviello, Ramachandrans. Vasan, et al. Sex hormone-binding globulin, but not testosterone, is associated prospectively and independently with incident metabolic syndrome in men: the framingham heart study. Diabetes Care 2011;34(11):2464-70.

17. Lopez-Miranda J, Perez-Martinez P. It is time to define metabolically obese but normal-weight (MONW) individuals. Clin Endocrinol (Oxf) 2013;79:314-5.

18. Martini AC, Molina RI, Ruiz RD, Fiol de Cuneo M. Obesity and male fertility. Rev Fac Cien Med Univ Nac Cordoba 2012;69(2).

19. Lotti, F., Rastrelli, G., Maseroli, E., Cipriani, S., Guaraldi, F., Krausz, C., et al. Impact of Metabolically Healthy Obesity in Patients with Andrological Problems. The Journal of Sexual Medicine 2019;16:821-832.

20. Wang C, Nieschlag E, Swerdloff R, Behre HM, Hellstrom WJ, Gooren LJ, et al., "Investigation, treatment, and monitoring of late-onset hypogonadism in males: ISA, ISSAM, EAU, EAA, and ASA recommendations," Journal of Andrology 2009;30:1-9.

21. Expert Panel on Detection E, Treatment of High Blood Cholesterol in A Executive Summary of The Third Report of The National Cholesterol Education Program (NCEP) Expert Panel on Detection, Evaluation, And Treatment of High Blood Cholesterol In Adults (Adult Treatment Panel III). JAMA $2001 ; 285: 2486-2497$. 
22. Pitteloud, N., Hardin, M., Dwyer, A.A., Valassi, E., Yialamas, M., Elahi, D., et al. Increasing insulin resistance is associated with a decrease in Leydig cell testosterone secretion in men. Clin. Endocrinol. Metab. 2005;90:2636-2641.

23. Lin T, Wang D, Stocco DM. Interleukin-1 inhibits Leydig cell steroidogenesis without affecting steroidogenic acute regulatory protein messenger ribonucleic acid or protein levels. $J$ Endocrinol 1998; $156: 461-467$.

24. Primeau V, Coderre L, Karelis AD, Brochu M, Lavoie ME, Messier V, et al. Characterizing the profile of obese patients who are metabolic healthy. Int I Obes (Lond) 2011; 35:971-81.

25. Wang, B., Zhang, M., Wang, S., Wang, C., Wang, J., Li, L., et al. Dynamic status of metabolically healthy overweight/obesity and metabolically unhealthy and normal weight and the risk of type 2 diabetes mellitus: A cohort study of a rural adult Chinese population. Obesity Research \& Clinical Practice 2018;12(1):61-71.

26. Ortega FB, Cadenas-Sanchez C, Migueles JH, Labayen I, Ruiz JR, Sui X, et al. Role of physical activity and fitness in the characterization and prognosis of the metabolically healthy obesity phenotype: a systematic review and meta-analysis. Prog Cardiovasc Dis 2018;61(2):190-205.

27. Yeh TL, Chen HH, Tsai SY, Lin CY, Liu SJ, Chien KL. The relationship between metabolically healthy obesity and the risk of cardiovascular disease: a systematic review and meta-analysis. J Clin Med 2019;8(8).

28. Perez-Martinez P, Alcala-Diaz JF, Delgado-Lista J, Garcia-Rios A, Gomez-Delgado F, Marin-Hinojosa C, et al. Metabolic phenotypes of obesity influence triglyceride and inflammation homoeostasis. European Journal of Clinical Investigation 2014; 44 (11): 1053-1064.

29. Wildman RP, Muntner P, Reynolds K, McGinn AP, Rajpathak S, Wylie-Rosett J et al. The obese without cardiometabolic risk factor clustering and the normal weight with cardiometabolic risk factor clustering: prevalence and correlates of 2 phenotypes among the US population (NHANES 19992004). Arch Intern Med 2008;168: 1617-1624.

30. Karelis AD, St-Pierre DH, Conus F, Rabasa-Lhoret R, Poehlman ET. Metabolic and body composition factors in subgroups of obesity: what do we know? J Clin Endocrinol Metab 2004;89:2569e75.

31. Yoo HJ, Hwang SY, Hong HC, Choi HY, Seo JA, Kim SG, et al. Association of metabolically abnormal but normal weight (MANW)and metabolically healthy but obese (MHO) individuals with arterial stiffness and carotid atherosclerosis. Atherosclerosis 2014;234(1), 218-223.

32. Choi KM, Cho HJ, Choi HY, Yang SJ, Yoo HJ, Seo JA et al. Higher mortality in metabolically obese normal-weight people than in metabolically healthy obese subjects in elderly Koreans. Clin Endocrinol (Oxf) 2013; 79: 364-370.

33. Kim, M., Paik, J.K., Kang, R. Kim, S.Y., Lee, S.H., Lee, J.H., et al. Increased oxidative stress in normalweight postmenopausal women with metabolic syndrome compared with metabolically healthy overweight/obese individuals. Metabolism 2012;62(4):554-560.

34. Vermeulen A, Kaufman J.M., Deslypere J.P., Thomas G. Attenuated luteinizing hormone (LH) pulse amplitude but normal LH pulse frequency, and its relation to plasma androgens in hypogonadism of 
obese men. The Journal of clinical endocrinology and metabolism. 1993; 76(5):1140-6.

35. George JT, Millar RP, Anderson RA. Hypothesis: kisspeptin mediates male hypogonadism in obesity and type 2 diabetes. Neuroendocrinology. 2010; 91(4):302-7.

\section{Tables}

Table 1 Baseline characteristics in different metabolic obesity phenotypes. MHNW: metabolically healthy normal weight; MHO: metabolically healthy obese; MUNW: metabolically unhealthy normal weight; MUO: metabolically unhealthy obese; BMI: body mass index; WC: waist circumference; TC: total cholesterol; TG: triglyceride; LDL-C: low density lipoprotein cholesterol; HDL-C: high density lipoprotein cholesterol; FBG: fasting blood glucose; 2hBG: 2-hour blood glucose; HbA1c: glycosylated hemoglobin; LH: luteinizing hormone; FSH: follicle-stimulating hormone; TT: total testosterone; SBP: systolic blood pressure; DBP: diastolic blood pressure; $\mathrm{DM}$ : diabetes mellitus; $\mathrm{CHD}$ : coronary heart disease. ${ }^{*} \mathrm{P}<0.05$, compared to MHNW; $₫ \mathrm{P}<0.05$, compared to $\mathrm{MHO}, \# \mathrm{P}<0.05$, compared to $\mathrm{MUNW}$; $£ \mathrm{P}<0.05$, compared to MUO. 


\begin{tabular}{|c|c|c|c|c|}
\hline Characteristics & $\begin{array}{l}\text { MHNW } \\
(n=1790)\end{array}$ & $\begin{array}{l}\text { MHO } \\
(n=1238)\end{array}$ & $\begin{array}{l}\text { MUNW } \\
(n=278)\end{array}$ & $\begin{array}{l}\text { MUO } \\
(n=775)\end{array}$ \\
\hline Age & $57.25 \pm 8.69^{\square £}$ & $54.66 \pm 8.59^{* \#}$ & $56.24 \pm 8.36^{\square f}$ & $54.01 \pm 8.80^{\star \#}$ \\
\hline BMI & $22.19 \pm 1.92^{\square \# £}$ & $27.51 \pm 2.61^{\star \# £}$ & $23.04 \pm 1.54^{\star \llbracket f}$ & $28.69 \pm 2.55^{\star \# \# ~}$ \\
\hline WC & $83.29 \pm 7.86^{\square \# £}$ & $92.61 \pm 7.54^{\star} £$ & $88.44 \pm 10.47^{\star £}$ & $98.71 \pm 9.39^{* \square \#}$ \\
\hline TC & $4.93 \pm 1.05^{\square \# £}$ & $5.06 \pm 1.06^{\star} f$ & $5.14 \pm 1.46^{*}$ & $5.26 \pm 1.30^{\star \square}$ \\
\hline TG & $1.07 \pm 0.67^{\square \# £}$ & $1.39 \pm 1.04^{\star \# £}$ & $2.47 \pm 1.71^{\star \star ⿷}$ & $2.72 \pm 2.19^{\star} \#$ \\
\hline LDL-C & $2.89 \pm 0.83^{\square £}$ & $3.12 \pm 0.86^{* \#}$ & $2.89 \pm 1.10^{\square £}$ & $3.05 \pm 0.94^{* \#}$ \\
\hline HDL-C & $1.52 \pm 0.39^{\square \# £}$ & $1.36 \pm 0.31^{* \# £}$ & $1.28 \pm 0.42^{* \llbracket £}$ & $1.19 \pm 0.29^{\text {*\#\# }}$ \\
\hline ALT & $17.84 \pm 9.47^{\square £}$ & $21.44 \pm 11.32^{\star} f$ & $20.24 \pm 10.28^{\star} £$ & 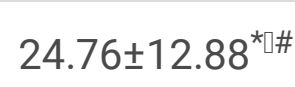 \\
\hline AST & $22.92 \pm 8.53^{\# £}$ & $23.32 \pm 8.66^{\#}$ & $25.04 \pm 12.00^{\star \square}$ & $23.87 \pm 8.92^{*}$ \\
\hline GGT & $29.96 \pm 35.61^{\square \# f}$ & $36.52 \pm 34.44^{\star \# £}$ & $59.32 \pm 71.50^{\star \Delta}$ & $53.81 \pm 52.10^{\star \Delta}$ \\
\hline FBG & $6.23 \pm 1.97^{\# £}$ & $6.15 \pm 1.54^{\# £}$ & $7.20 \pm 2.42^{*[}$ & $7.25 \pm 2.19^{\star[}$ \\
\hline $2 \mathrm{hBG}$ & $8.85 \pm 4.53^{\# £}$ & $8.78 \pm 4.12^{\# £}$ & $11.71 \pm 5.69^{\star \square}$ & $11.51 \pm 5.26^{\text {* }}$ \\
\hline $\mathrm{HbA1C}$ & $6.01 \pm 1.23^{\# £}$ & $5.98 \pm 0.95^{\# £}$ & $6.41 \pm 1.46^{*[}$ & $6.53 \pm 1.26^{\text {* }}$ \\
\hline FSH & $11.69 \pm 11.71^{\square f}$ & $10.06 \pm 8.61^{*}$ & $11.01 \pm 11.86$ & $9.76 \pm 9.09^{*}$ \\
\hline LH & $8.09 \pm 5.83^{\square £}$ & $6.76 \pm 4.78^{*}$ & $7.78 \pm 5.38^{\square f}$ & $6.44 \pm 4.21^{\star \#}$ \\
\hline TT & $21.92 \pm 7.53^{\square \# £}$ & $17.77 \pm 6.05^{\star} £$ & $17.26 \pm 6.28^{\star} f$ & $14.79 \pm 5.19^{\star \# \#}$ \\
\hline SBP & $137.59 \pm 20.67^{\square \# 1}$ & $140.50 \pm 20.58^{\star \# £}$ & $150.11 \pm 18.55^{\star \square}$ & $147.40 \pm 17.97^{\star \text { « }}$ \\
\hline DBP & $80.13 \pm 12.63^{\square \# £}$ & $81.61 \pm 13.88^{\star \# £}$ & $86.89 \pm 10.35^{\star \llbracket f}$ & $88.17 \pm 11.28^{\star \square \#}$ \\
\hline DM (\%) & $7.54^{\# £}$ & $8.00^{\# £}$ & $12.59^{\star \square}$ & $16.52^{\star \square}$ \\
\hline Hypertension (\%) & $12.78^{\square \nsubseteq £}$ & $20.06^{\star} f$ & $22.08^{\star} £$ & 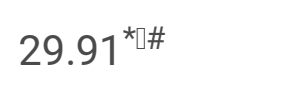 \\
\hline CHD (\%) & $5.49^{f}$ & $6.61^{£}$ & 8.70 & $9.95^{\star}$ \\
\hline smoking status (\%) & $\square £$ & *\# & 口 & * \\
\hline never & 35.43 & 39.64 & 33.79 & 35.88 \\
\hline ever & 15.44 & 19.25 & 15.53 & 20.07 \\
\hline
\end{tabular}




\begin{tabular}{|lllll|} 
current & 49.13 & 41.12 & 50.68 & 44.05 \\
$\begin{array}{l}\text { Alcohol consumption } \\
(\%)\end{array}$ & & & & \\
never & 24.79 & 23.31 & 26.99 & 21.73 \\
ever & 12.96 & 10.63 & 11.50 & 11.88 \\
current & 62.25 & 66.06 & 61.50 & 66.38 \\
\hline
\end{tabular}

MHNW: metabolically healthy normal weight; MHO: metabolically healthy obese; MUNW: metabolically unhealthy normal weight; MUO: metabolically unhealthy obese; BMI: body mass index; WC: waist circumference; TC: total cholesterol; TG: triglyceride; LDL-C: low density lipoprotein cholesterol; HDL-C: high density lipoprotein cholesterol; FBG: fasting blood glucose; 2hBG: 2-hour blood glucose; HbA1c: glycosylated hemoglobin; LH: luteinizing hormone; FSH: follicle-stimulating hormone; TT: total testosterone; SBP: systolic blood pressure; DBP: diastolic blood pressure; DM: diabetes mellitus; CHD: coronary heart disease.

${ }^{*} \mathrm{P}<0.05$, compared to MHNW; ${ }^{\square} \mathrm{P}<0.05$, compared to $\mathrm{MHO},{ }^{\#} \mathrm{P}<0.05$, compared to MUNW; ${ }^{£} \mathrm{P}<0.05$, compared to MUO.

Table 2 Separate and mutually adjusted correlations of BMI and presence of the MS with total testosterone. BMI: body mass index; MS: metabolic syndrome; TT: total testosterone; LH: luteinizing hormone. Adjusted for age, LH, smoking status (never, ever, current), alcohol intake (never, ever, current).

Separate

\begin{tabular}{|lllll|}
\hline BMI & TT $<12 \mathrm{nmol} / \mathrm{L}$ & TT $>12 \mathrm{nmol} / \mathrm{L}$ & OR & $95 \% \mathrm{Cl}$ \\
\hline$<25.0$ & 157 & 1911 & 1.000 & Ref \\
\hline$\geq 25.0$ & 406 & 1607 & 3.072 & $2.414-3.911$ \\
\hline MS & & & & \\
\hline No & 293 & 2735 & 1.000 & Ref \\
\hline Yes & 270 & 783 & 3.294 & $2.631-4.125$ \\
\hline
\end{tabular}

Adjusted for age, LH, smoking status (never, ever, current), alcohol intake (never, ever, current).

Mutually 


\begin{tabular}{|lllll|}
\hline BMI & TT $<12 \mathrm{nmol} / \mathrm{L}$ & TT $>12 \mathrm{nmol} / \mathrm{L}$ & OR & $95 \% \mathrm{Cl}$ \\
\hline$<25.0$ & 157 & 1911 & 1.000 & Ref \\
\hline$\geq 25.0$ & 406 & 1607 & 2.409 & $1.873-3.097$ \\
\hline MS & & & & \\
\hline No & 293 & 2735 & 1.000 & Ref \\
\hline Yes & 270 & 783 & 2.618 & $2.073-3.308$ \\
\hline
\end{tabular}

BMI: body mass index; MS: metabolic syndrome; TT: total testosterone; LH: luteinizing hormone.

Adjusted for age, LH, smoking status (never, ever, current), alcohol intake (never, ever, current).

Table 3 Logistic regression analysis of hypotestosteronemia according to different metabolic phenotypes of obesity. MHNW: metabolically healthy normal weight; MHO: metabolically healthy obese; MUNW: metabolically unhealthy normal weight; MUO: metabolically unhealthy obese. Model 1: unadjusted; Model 2: adjusted for age; Model 3: adjusted for age, LH, smoking status and alcohol consumption.

\begin{tabular}{|llll|}
\hline & Model 1 & Model 2 & Model 3 \\
\hline MHNW & Ref. & Ref. & Ref. \\
\hline MHO & $2.736(2.132-3.512)$ & $2.606(2.027-3.349)$ & $2.540(1.868-3.453)$ \\
\hline MUNW & $3.332(2.314-4.799)$ & $3.279(2.276-4.726)$ & $2.931(1.890-4.548)$ \\
\hline MUO & $6.213(4.842-7.972)$ & $5.861(4.558-7.535)$ & $6.370(4.697-8.638)$ \\
\hline
\end{tabular}

MHNW: metabolically healthy normal weight; MHO: metabolically healthy obese; MUNW: metabolically unhealthy normal weight; MUO: metabolically unhealthy obese.

Model 1: unadjusted; Model 2: adjusted for age; Model 3: adjusted for age, LH, smoking status and alcohol consumption.

\section{Figures}



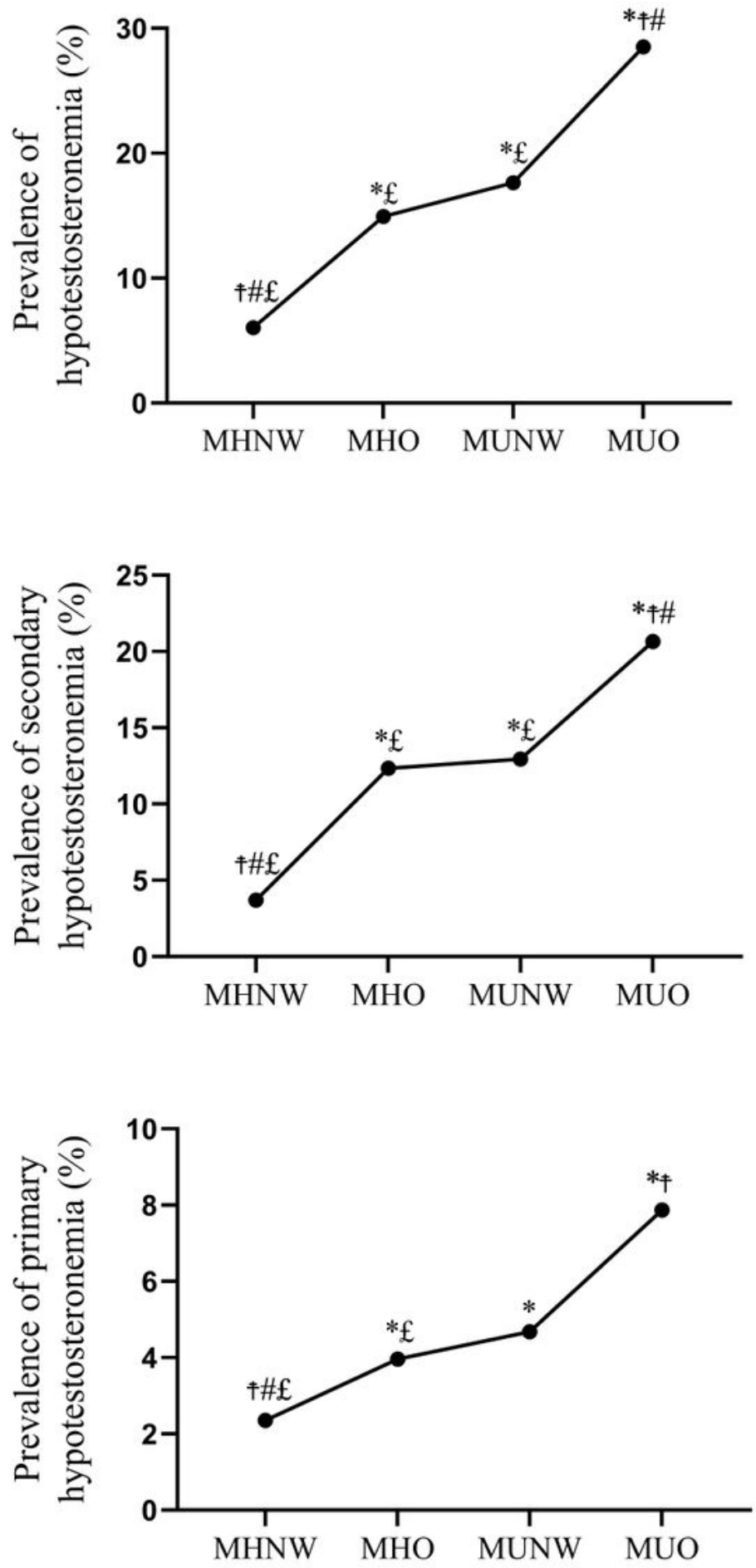

\section{Figure 1}

Prevalence of hypotestosteronemia in different metabolic phenotypes of obesity. metabolically healthy normal weight (MHNW); metabolically healthy obese (MHO); metabolically unhealthy normal weight (MUNW) and metabolically unhealthy obese (MUO). ${ }^{*} \mathrm{P}<0.05$, compared to MHNW group; $\mathbb{P}<0.05$, compared to $\mathrm{MHO}$ group; $\# \mathrm{P}<0.05$, compared to MUNW group; $£ \mathrm{P}<0.05$, compared to MUO group. (Fig $1 \mathrm{~s}$ used graph pad prism8 to create the artwork). 